\title{
Modeling the Effect of Asymptomatic Cases, Social Distancing, and Lockdowns in the First and Second waves of the COVID-19 Pandemic: A Case Study of Italy
}

\author{
Muhamad Khairulbahri ${ }^{1 *}$ \\ ${ }^{1}$ The Graduate Programme of Development Studies, (Jurusan Pascasarjana Studi Pembangunan), Bandung Institute of Technology, \\ 40132, Indonesia.
}

Received 09 February 2021; Revised 03 June 2021; Accepted 20 June 2021; Published 01 September 2021

\begin{abstract}
The SEIR model of COVID-19 is developed to investigate the roles of physical distancing, lockdowns, and asymptomatic cases in Italy. In doing so, two types of policies including behavioral measures and lockdown measures are embedded in the model. Compared with existing models, the model successfully reproduces similar multiple observed outputs such as infected and recovered patients in Italy by July 2020. This study concludes that the first policy is important once the number of infected cases is relatively low. However, once the number of infected cases is too high, so the society cannot identify infected and disinfected people, the second policy must be applied soon. It is thus this study suggests that relaxed lockdowns lead to the second wave of the COVID-19 around the world. It is hoped that the model can enhance our understanding of the roles of behavioral measures, lockdowns, and undocumented cases, so-called asymptomatic cases, on the COVID-19 flow.
\end{abstract}

Keywords: COVID-19; SEIR Model; System Dynamics; Behavioral Measures; Lockdowns.

\section{Introduction}

Despite its high quality of the national healthcare program, Italy was the epicenter of the coronavirus disease 2019 (the COVID-19) during the European first wave. The Italy first closure owing to COVID-19 was on February 22nd, 2020. As infected cases spiked, the government started the first measure on March 4th by closing public places such as stores, and schools as well as enforcing physical distancing in severely affected areas close to Milan and Venice [1]. This lockdown was lengthened on March 9th, owing to a rise of documented cases and confirmed deaths at about 7,000 people and 400 people respectively.

Some existing studies discussed issues relating to Italian COVID-19 cases such as policy measures [1-3], the dynamics of high COVID-19 cases [4-9], and comparisons of the COVID-19 in Italy and China [10]. It appears that existing studies have successfully modeled the COVID-19 cases in Italy and analyzed the main factors of Italian COVID-19 flow. However, to the best author's knowledge, no available study has successfully modeled two different notified policies in tackling the COVID-19: the behavior policy and the lockdown policy. This is important as other studies [11-14] highlighted the importance of the first and the second policies in minimizing the COVID-19 flow in Italy. Hence, this study compares the efficacy of the two different measures in the Italian COVID-19 cases.

\footnotetext{
* Corresponding author: databahri@yahoo.com

doi http://dx.doi.org/10.28991/SciMedJ-2021-0303-8

$>$ This is an open access article under the CC-BY license (https://creativecommons.org/licenses/by/4.0/).

(C) Authors retain all copyrights.
} 
This study also investigates three important cases simultaneously including recovered, infected, and death cases, especially during the first wave in Italy. As some studies mentioned [2-4, 6] possible roles of undocumented cases, this study also investigates the role of the undocumented cases in Italy. With keep these points in mind, the author believes that modeling the COVID-19 in Italy can give us an understanding of the dynamic flow of the COVID-19 in terms of behavior, lockdown policies, and undocumented cases. Moreover, this study offers a more comprehensive analysis as this study analyzes the quantitative impacts of undocumented cases, the quantitative impacts of behavioral measures, and the quantitative impacts of lockdowns than existing studies $[1,15,16]$.

In the first section, this study explains existing studies of COVID-19 in Italy. Furthermore, this study explains the system dynamics approach to understand the dynamic of susceptible, exposed, infectious, and recovered cases in Italy. The system dynamics approach has a long tradition to model health issues such as epidemiology, healthcare facilities, and non-communicable diseases $[17,18]$. Because of this, this study develops the Italian SEIR model based on the system dynamics approach to simulate deaths, recoveries, and incidences in Italy.

\section{Materials and Methods}

Required data were collected from https://www.worldometers.info/coronavirus/, www.rki.de (Robert Koch Institute), and https://coronavirus.jhu.edu (Johns Hopkins University). Three data types including infected, death, recovered cases were extracted from the aforementioned sources. Previous studies based on the system dynamics approach have successfully simulated infectious, non-infectious diseases, and other healthcare issues [17-19]. Owing to this, the SEIR model used in this study is developed based on the system dynamics approach. Despite the usefulness of the SEIR model, some uncertain parameters have not been defined or unknown. To obtain the best parameter values, this study applies the Markov Chain Monte Carlo (MCMC) calibration owing to unknown parameters using built functions in Vensim@

The model starts the simulation from January $1^{\text {st }}, 2020$, and runs on daily terms until the end of July 2020. Simulated parameters such as an incubation time, Ro, and the infection duration are based on existing studies. It is important to note that the SEIR model will be set in two types of the first confirmed cases. Usuelli (2020) noted that COVID-19 models of Italy should assume that the first confirmed case is infected Chinese tourists who were acknowledged as imported COVID-19 cases on January 31 $1^{\text {st }}, 2020$ [20]. While other studies De Natale et al., Gatto et al. and Sjödin et al. (2020) assumed that the first Italian confirmed case should be the first infected male in February $21^{\text {st }}, 2020[1,4,6]$. By comparing two types of the first confirmed cases, this study wants to prove the importance of behavioral measures such as physical distancing and handwashing. The logic is if there are not significant differences of estimated parameter values between the two first confirmed cases, this intuitively means that behavioral measures were effective in hindering the COVID-19 flow from January $31^{\text {st }}, 2020$ to February $21^{\text {st }}, 2020$.

\section{Results and Discussion}

To obtain the best value for each parameter, at the first step, the SDM is embedded by range values stated in Table 1 and at the second step, we run the Markov Chain Monte Carlo (MCMC) calibration, available in Vensim $\odot$ [21]. To accommodate uncertain parameter values, this study set range values based on existing studies. For instance, as other studies, this study sets Ro ranges between 3 and 4 [22, 23].

Table 1. Parameter values of the SDM. Bracketed values are min and max values based on existing studies

\begin{tabular}{|c|c|c|c|}
\hline No. & Names & Values & References \\
\hline 1 & The two first confirmed cases & January $31^{\text {st }}, 2020$; February $21^{\text {st }}, 2020$ & $\begin{array}{l}\text { De Natale et al.; Goumenou et al.; and Raheem } \\
(2020)[4,7,16]\end{array}$ \\
\hline 2 & Ro (basic reproduction number) & $3.5(3-4)$ & Romano; Romano et al. $(2020)[22,23]$ \\
\hline 3 & Incubation time & 4 days (3-5) days & $\begin{array}{l}\text { Lauer et al.; Sjödin et al.; and Yu et al. (2020) } \\
{[1,24,25]}\end{array}$ \\
\hline 4 & Infection duration & $26(7-45)$ days & ISS $(2020)[26]$ \\
\hline 5 & Recovery time & $8(5-12)$ days & Grasselli et al. (2020) [8] \\
\hline 6 & $\begin{array}{l}\text { Behavioral reaction time } \\
\text { The behavioral reduction time is measured } \\
\text { between the first infection case and the } \\
\text { beginning of the behavioral risk reduction } \\
\text { actions. This time measurement also applies } \\
\text { to other time measurements. }\end{array}$ & $\begin{array}{l}15 \text { days on March } 4^{\text {th }} \text { (public place closures) and } 22^{\text {nd }} \\
\text { (the national lockdown). } \\
\text { There was also the lockdown on March } 8^{\text {th }} \text { (The } \\
\text { lockdown of Northern Italy) }\end{array}$ & Goumenou et al.; and ISS $(2020)[7,26]$ \\
\hline 7 & $\begin{array}{l}\text { Behavioral risk reduction } \\
\text { Lockdown reaction time }\end{array}$ & $\begin{array}{l}\text { This policy is voluntary acts without legal enforcement. } \\
\text { This is assumed to be between } 10 \text { and } 50 \%\end{array}$ & \\
\hline 8 & $\begin{array}{l}\text { As there are many lockdowns, another } \\
\text { variable i.e., "delay time" (1-5 days) is } \\
\text { inserted in the SEIR model. }\end{array}$ & 45 days (after the first case) & ISS (2020) [26] \\
\hline 9 & Lockdown risk reduction & $\begin{array}{l}\text { The lockdowns are very strict and enforced by law, so } \\
\text { the efficacy is high }(60-95 \%)\end{array}$ & \\
\hline 10 & $\begin{array}{l}\text { Fraction of undocumented cases upon } \\
\text { documented cases. }\end{array}$ & $55 \%(46-62) \%$ & $\begin{array}{l}\text { Flaxman et al.; Lavezzo et al.; Gatto et al.; and } \\
\text { Li et al. (2020) }[2,3,6,27]\end{array}$ \\
\hline
\end{tabular}




\subsection{The SEIR Model}

The system dynamics model (SDM), so-called stock-flow model, for Italian cases can be seen in Figure 1. The SDM consists of five stocks such as the susceptible, infected, and recovered cases. The SDM also accommodates the impacts of behavioral measures such as physical distancing, handwashing, and mask covers. For these measures, the SDM applies two variables namely, "behavioral risk reduction" and "behavioral reaction time". These two variables respectively explain the efficacy of and starting time of the aforementioned measures in minimizing the flow of COVID-19. The more striking COVID-19 cases, the more critical or defensive measures are taken such as isolating patients and lockdowns. In considering striking cases, the SDM also captures the impacts of lockdowns through two variables: "lockdown risk reduction" and "lockdown reduction time". Similarly, the two variables respectively explain the efficacy and starting time of lockdown policies in minimizing the flow of COVID-19. The SEIR model is sketched as seen in Figure 1.

Other studies $[3,7,28]$ stated that undocumented cases may be responsible for rising cases in Italy and other cities in China [27]. The SDM reveals the roles of undocumented cases through two variables: "undocumented infected cases" and "fraction of undocumented cases". A fraction of undocumented cases is a portion of undocumented cases compared with documented cases. In this model, documented cases are represented as "infected rates".
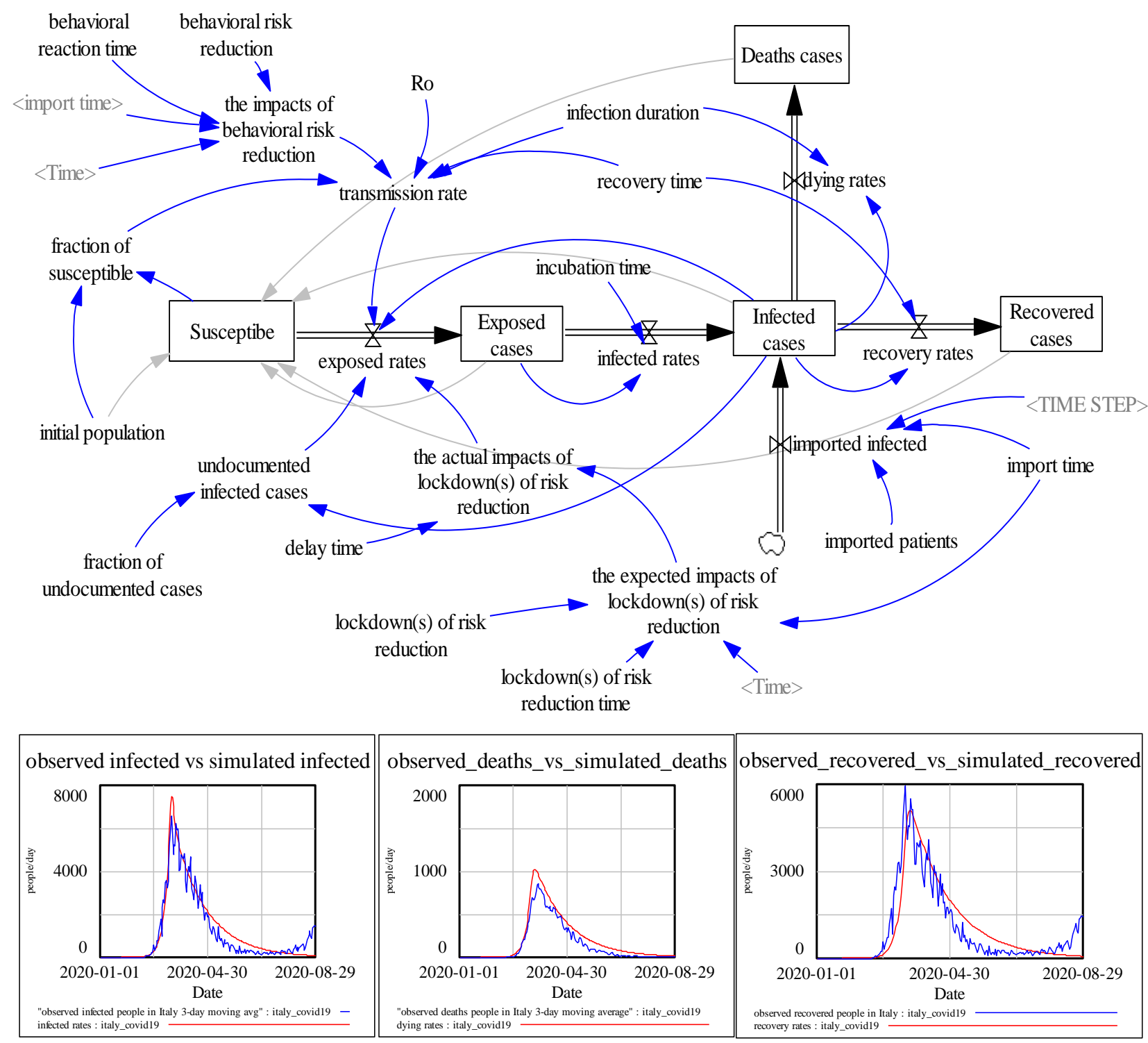

Figure 1. The SIER model for Italy

As previously mentioned, the SEIR model separates patients into four categories, exposed, infected, death, recovered, and undocumented cases. In the model, undocumented cases are represented as a multiplication between a fraction of undocumented cases and infected cases. Time variables such as recovery time are defined as the average time between the symptom onset and recoveries while infected duration is defined as the average time between the symptom onset and deaths. 
The SEIR model calculates the number of infected cases, deaths, and recoveries based on Equations 1 to 3:

infected rates $=$ "exposed cases"/incubation time

dying rates $=$ "infected cases"/infection duration

recovery rates $=$ "infected cases"/recovery time

The transmission rate measures the number of exposed people after contacting or stand closes with infected people. Following Fiddaman (2020) [29], the transmission rate is calculated based on Equation 3. For the first policy, its impact is calculated based on Equation 4. Equation 4 means that the first policy of behavioral reduction risk decreases transmission rate based on two factors: "behavioral reduction risk" and "behavioral reduction time".

Transmission rate $=($ Ro/recovery time $) \times$ fraction of susceptible $\times(1$ the impacts of behavioral risk reduction $)$

The second policy i.e. lockdowns are calculated similarly to Equation 4. Equations 5a and 5b show the number of exposed cases decreases after the second policy starts at "lockdown reduction time".

The expected impacts of lockdown risk reduction = IF THEN ELSE (Time>=import time + "lockdown risk reduction time", "lockdown risk reduction", 0)

The actual impacts of lockdown risk reduction = DELAY3I ("the expected impacts of lockdown risk reduction", delay time, "the expected impacts of lockdown risk reduction")

After running the MCMC calibration process, optimized values for each parameter are shown in Table 2. The second and third columns represent optimized values if the first case is around January and around February 2020 respectively. As seen in Table 2, there are no significant differences between the two types of the first confirmed cases. This implies that the first policy i.e., voluntary acts such as social distancing and handwashing hindered the COVID-19 in early January 2020. In other words, the first policy was effective from January $31^{\text {st }}, 2020$ to February $21^{\text {st }}, 2020$.

This study also shows that the first policy is helpful once the number of infected cases is relatively low [30]. However, that study [30] also claimed that once the COVID-19 cases rise significantly so separating infected and disinfected people owing to is relatively difficult, policymakers should apply the second policy.

Table 2. The best parameter values. Variables with an asterisk should be quantified from the first confirmed case

\begin{tabular}{clcc}
\hline No. & \multicolumn{1}{c}{ Variables } & Estimated values \#1 & Estimated values \#2 \\
\hline 1 & Ro (basic reproduction number) & $3.68(3-3.7)$ & $3.7(3-3.7)$ \\
2 & Incubation time (days) & $3(3-5)$ days & $3(3-5)$ days \\
3 & Infection duration (days) & $16.5(7-45)$ days & $12.56(7-45)$ days \\
4 & Recovery time (days) & $5.7(3-35)$ days & $2.75(3-35)$ days \\
5 & Fraction of undocumented cases & $73 \%(46-62) \%$ & $79 \%(46-62) \%$ \\
6 & The behavioral reaction time (days) & 37.87 days* & 20.7 days* \\
7 & The behavioral risk reduction & $37.33 \%$ & $31.37 \%$ \\
8 & The lockdown(s) of risk reduction time & 33 days* & 18 days* \\
9 & The lockdown(s) of risk reduction & $65 \%$ & $65 \%$ \\
10 & Delay time (days) & 5 days & 5.3 days \\
11 & Import time & $\begin{array}{l}31 \text { days (the first confirmed cases } \\
\text { should be a case announced on }\end{array}$ & $\begin{array}{l}52 \text { days (the first confirmed case was } \\
\text { February } 21^{\text {st }} \text { or day } 52 \text { from January }\end{array}$ \\
\hline
\end{tabular}

Looking back at Table 2, it appears that voluntary acts such as handwashing and social distancing, despite their importance in hindering the COVID-19 flow, have relatively lower effects than the lockdown policy (31-37\% vs. $65 \%$ ). However, this does not mean that voluntary acts are not needed. The first policy such as handwashing and social distancing could hinder the flow of COVID-19 in the first wave despite its relatively low effects.

This study also highlights that the important roles of undocumented cases, so-called asymptomatic cases, during the first wave of COVID-19 in Italy. This study finds that undocumented case was about $70 \%$ of the confirmed cases in Italy. Moreover, the SEIR model can reproduce similar outputs compared to respective observed outputs as seen in Figure 1. The SD model has symmetric Mean Percentage Errors (sMAPE) less than $10 \%$ as seen in Table 3. 
Table 3. sMAPE for the SEIR model

\begin{tabular}{cc}
\hline Variables & SMAPE \\
\hline Infected cases & $<10 \%$ \\
Death cases & $<10 \%$ \\
Recovered cases & $<10 \%$ \\
\hline
\end{tabular}

\section{Conclusion}

Using the system dynamics approach, Italian COVID-19 is successfully modeled. Differing from other studies, this study aims to model dynamics of infected, recovered, and death cases incorporating undocumented cases. Moreover, the SDM captures the positive effects of policy measures including behavioral policies (wear masks, handwashing, and social distancing) and lockdowns or isolations

Once optimized values for each parameter are obtained through the MCMC calibration, the SEIR successfully reproduces similar outputs as observed outputs. The SEIR model also provides evidence that the first policy has relatively lower impacts than the second policy. Nevertheless, the first policy is important once the number of infected cases is relatively low. Once the number of infected cases is high, the second policy shows relatively higher impacts in minimizing the flow of COVID-19. The higher impacts of the second policy than the first policy may be a clue to the second wave of the COVID-19 around the world.

\subsection{Supplemental Materials}

- The SEIR model is available online at: https://osf.io/vuypf;

- Appendix I presents the results of sensitivity analysis.

\section{Declarations}

\subsection{Funding}

The author received no financial support for the research, authorship, and/or publication of this article.

\subsection{Acknowledgements}

The author expresses his gratitude to all supportive colleagues from Bandung Institute of Technology.

\subsection{Ethical Approval}

The manuscript does not contain experiments on animals and humans; hence ethical permission was not required.

\subsection{Data Availability Statement}

Publicly available datasets were analyzed in this study. This data can be found here:

https://www.worldometers.info/coronavirus/, www.rki.de (Robert Koch Institute), and https://coronavirus.jhu.edu (Johns Hopkins University).

\subsection{Conflict of Interest}

The author declares that he has not known competing financial interests or personal relationships that could have appeared to influence the work reported in this paper.

\section{References}

[1] Sjödin, H., Wilder-Smith, A., Osman, S., Farooq, Z., \& Rocklöv, J. (2020). Only strict quarantine measures can curb the coronavirus disease (COVID-19) outbreak in Italy, 2020. Eurosurveillance, 25(13). doi:10.2807/15607917.es.2020.25.13.2000280.

[2] Flaxman, S., Mishra, S., Gandy, A., Unwin, H. J. T., Coupland, H., Mellan, T. A., ... \& Bhatt, S. (2020). Estimating the number of infections and the impact of non-pharmaceutical interventions on COVID-19 in European countries: technical description update. arXiv:2004.11342.

[3] Lavezzo, E., Franchin, E., Ciavarella, C., Cuomo-Dannenburg, G., ... Barzon, L. (2020). Suppression of a SARS-CoV-2 outbreak in the Italian municipality of Vo'. Nature, 584(7821), 425-429. doi:10.1038/s41586-020-2488-1.

[4] De Natale, G., Ricciardi, V., De Luca, G., De Natale, D., Di Meglio, G., Ferragamo, A., ... Troise, C. (2020). The COVID-19 Infection in Italy: A Statistical Study of an Abnormally Severe Disease. Journal of Clinical Medicine, 9(5), 1564. doi:10.3390/jcm9051564. 
[5] Garazzino, S., Montagnani, C., Donà, D., Meini, A., Felici, E., ... Vergine, G. (2020). Multicentre Italian study of SARS-CoV-2 infection in children and adolescents, preliminary data as at 10 April 2020. Eurosurveillance, 25(18). doi:10.2807/15607917.es.2020.25.18.2000600.

[6] Gatto, M., Bertuzzo, E., Mari, L., Miccoli, S., Carraro, L., Casagrandi, R., \& Rinaldo, A. (2020). Spread and dynamics of the COVID-19 epidemic in Italy: Effects of emergency containment measures. Proceedings of the National Academy of Sciences, 117(19), 10484-10491. doi:10.1073/pnas.2004978117.

[7] Goumenou, M., Sarigiannis, D., Tsatsakis, A., Anesti, O., Docea, A., Petrakis, D., ... Calina, D. (2020). COVID - 19 in Northern Italy: An integrative overview of factors possibly influencing the sharp increase of the outbreak (Review). Molecular Medicine Reports. doi:10.3892/mmr.2020.11079.

[8] Grasselli, G., Zangrillo, A., Zanella, A., Antonelli, M., Cabrini, L., Castelli, A., ... Fumagalli, R. (2020). Baseline Characteristics and Outcomes of 1591 Patients Infected With SARS-CoV-2 Admitted to ICUs of the Lombardy Region, Italy. JAMA, 323(16), 1574-1581. doi:10.1001/jama.2020.5394

[9] Grossi, U., Zanus, G., \& Felice, C. (2020). Coronavirus disease 2019 in Italy: The Veneto model. Infection Control \& Hospital Epidemiology, 42(2), 236-237. doi:10.1017/ice.2020.225.

[10] Nesteruk, I. (2020). Comparison of the coronavirus epidemic dynamics in Italy and mainland China. Preprint.] ResearchGate.

[11] Carlucci, L., D’Ambrosio, I., \& Balsamo, M. (2020). Demographic and Attitudinal Factors of Adherence to Quarantine Guidelines during COVID-19: The Italian Model. Frontiers in Psychology, 11. doi:10.3389/fpsyg.2020.559288.

[12] Graffigna, G., Bosio, C., Savarese, M., Barello, M., \& Barello, S. (2020). “\#I-Am-Engaged”: Conceptualization and First Implementation of a Multi-Actor Participatory, Co-designed Social Media Campaign to Raise Italians Citizens' Engagement in Preventing the Spread of COVID-19 Virus. Frontiers in Psychology, 11. doi:10.3389/fpsyg.2020.567101.

[13] Meier, K., Glatz, T., Guijt, M. C., Piccininni, M., van der Meulen, M., ... Atmar, K. (2020). Public perspectives on protective measures during the COVID-19 pandemic in the Netherlands, Germany and Italy: A survey study. PLOS ONE, 15(8), e0236917. doi:10.1371/journal.pone.0236917

[14] Pagnini, F., Bonanomi, A., Tagliabue, S., Balconi, M., Bertolotti, M., Confalonieri, E., ... Villani, D. (2020). Knowledge, Concerns, and Behaviors of Individuals during the First Week of the Coronavirus Disease 2019 Pandemic in Italy. JAMA Network Open, 3(7), e2015821. doi:10.1001/jamanetworkopen.2020.15821.

[15] Dehning, J., Zierenberg, J., Spitzner, F. P., Wibral, M., Neto, J. P., Wilczek, M., \& Priesemann, V. (2020). Inferring change points in the spread of COVID-19 reveals the effectiveness of interventions. Science, 369(6500), eabb9789. doi:10.1126/science.abb9789.

[16] Raheem, A. (2020). Estimating Cases of COVID-19 from Daily Death Data in Italy. medRxiv. doi:10.1101/2020.03.17.20037697.

[17] Darabi, N., \& Hosseinichimeh, N. (2020). System dynamics modeling in health and medicine: a systematic literature review. System Dynamics Review, 36(1), 29-73. doi:10.1002/sdr.1646.

[18] Homer, J. B., \& Hirsch, G. B. (2006). System Dynamics Modeling for Public Health: Background and Opportunities. American Journal of Public Health, 96(3), 452-458. doi:10.2105/ajph.2005.062059.

[19] Davahli, M. R., Karwowski, W., \& Taiar, R. (2020). A System Dynamics Simulation Applied to Healthcare: A Systematic Review. International Journal of Environmental Research and Public Health, 17(16), 5741. doi:10.3390/ijerph17165741.

[20] Usuelli, M. (2020). The Lombardy region of Italy launches the first investigative COVID-19 commission. The Lancet, 396(10262), e86-e87. doi:10.1016/s0140-6736(20)32154-1.

[21]Vensim user guide. (2020). Markov Chain Monte Carlo \& Simulated Annealing. Available online: https://www.vensim.com/documentation/mcmc_sa.html (accessed on February 2021).

[22] Romano, S., Fierro, A., \& Liccardo, A. (2020). Beyond the peak: A deterministic compartment model for exploring the Covid19 evolution in Italy. PloS One, 15(11), e0241951. doi:10.1371/journal.pone.0241951.

[23] Romano, S. (2020). Modeling the Covid-19 outbreak in Italy: estimates and forecasts. Università degli Studi di Napoli Federico II.

[24] Lauer, S. A., Grantz, K. H., Bi, Q., Jones, F. K., Zheng, Q., Meredith, H. R., ... Lessler, J. (2020). The Incubation Period of Coronavirus Disease 2019 (COVID-19) From Publicly Reported Confirmed Cases: Estimation and Application. Annals of Internal Medicine, 172(9), 577-582. doi:10.7326/m20-0504.

[25] Yu, P., Zhu, J., Zhang, Z., \& Han, Y. (2020). A Familial Cluster of Infection Associated With the 2019 Novel Coronavirus Indicating Possible Person-to-Person Transmission During the Incubation Period. The Journal of Infectious Diseases, 221(11), 1757-1761. doi:10.1093/infdis/jiaa077. 
[26] ISS. (Istituto Superiore di Sanità). (2020). Characteristics of SARS-CoV-2 Patients Dying in Italy. Available online: https://www.epicentro.iss.it/en/coronavirus/bollettino/Report-COVID-2019_20_april_2020.pdf (accessed on 9 August 2020).

[27] Li, R., Pei, S., Chen, B., Song, Y., Zhang, T., Yang, W., \& Shaman, J. (2020). Substantial undocumented infection facilitates the rapid dissemination of novel coronavirus (SARS-CoV-2). Science, 368(6490), 489-493. doi:10.1126/science.abb3221.

[28] Grossi, U., Zanus, G., \& Felice, C. (2021). Coronavirus disease 2019 in Italy: The Veneto model. Infection Control \& Hospital Epidemiology, 42(2), 236-237. doi:10.1017/ice.2020.225

[29] Fiddaman, T. (2020). 'A Community Coronavirus Model for Bozeman'. MetaSD (blog). Available online: https://metasd.com/2020/03/community-coronavirus-model-bozeman/ (accessed on July 2020).

[30] Piguillem, Facundo and Shi, Liyan, Optimal Covid-19 Quarantine and Testing Policies (April 2020). CEPR Discussion Paper No. DP14613.

[31] Maani., K. \& Cavana.R.Y. (2007) System Thinking, System Dynamics: Managing change and Complexity. Auckland, New Zealand: Pearson Education New Zealand. 


\section{Appendix I: Sensitivity Analysis}

Two steps of sensitivity analysis are conducted. The first sensitivity analysis is useful to categorize highly sensitive, very sensitive, and sensitive parameters [31] and the second sensitivity analysis is run for multiple varied given parameters, so-called Latin Hypercube Sampling (LHS). The two types of sensivity analysis are available in Vensim $\bigodot$.

Of the first sensitivity analysis, given parameters are firmly changed $\pm 10 \%$. If desired outputs including infected, death, and recovered cases are altered higher than $35 \%$ or less than $15 \%$, tested parameters are categorized as highly sensitive parameters or sensitive parameters respectively [31]. As seen in table A1, five parameters are highly sensitive such as Ro, infection duration, and incubation time. The others are relatively sensitive to given outputs.

Table A1. Sensitivity analysis for each uncertain parameter

\begin{tabular}{clc}
\hline No. & \multicolumn{1}{c}{ Variables } & Sensitivity results \\
\hline 1 & Ro (basic reproduction number) & $>35 \%$ \\
2 & Infection duration (days) & $>35 \%$ \\
3 & Incubation time (days) & $>35 \%$ \\
4 & Recovery time & $>35 \%$ \\
5 & Fraction of undocumented cases & $>35 \%$ \\
6 & Delay time (days) & $<15 \%$ \\
7 & The behavioral risk reduction & $<15 \%$ \\
8 & The behavioral reaction time (days) & $<15 \%$ \\
9 & The lockdown risk reduction & $<15 \%$ \\
10 & The lockdown risk reduction time (days) & $<15 \%$ \\
\hline
\end{tabular}

In the second step, sensitivity analysis is conducted after highly sensitive parameters are varied based on existing studies (Table 1). The results of the second sensitivity analysis are presented in figures A2. The colours show $50 \%$ (yellow), $75 \%$ (green), $95 \%$ (blue), and 100\% (grey) of the total sampling.
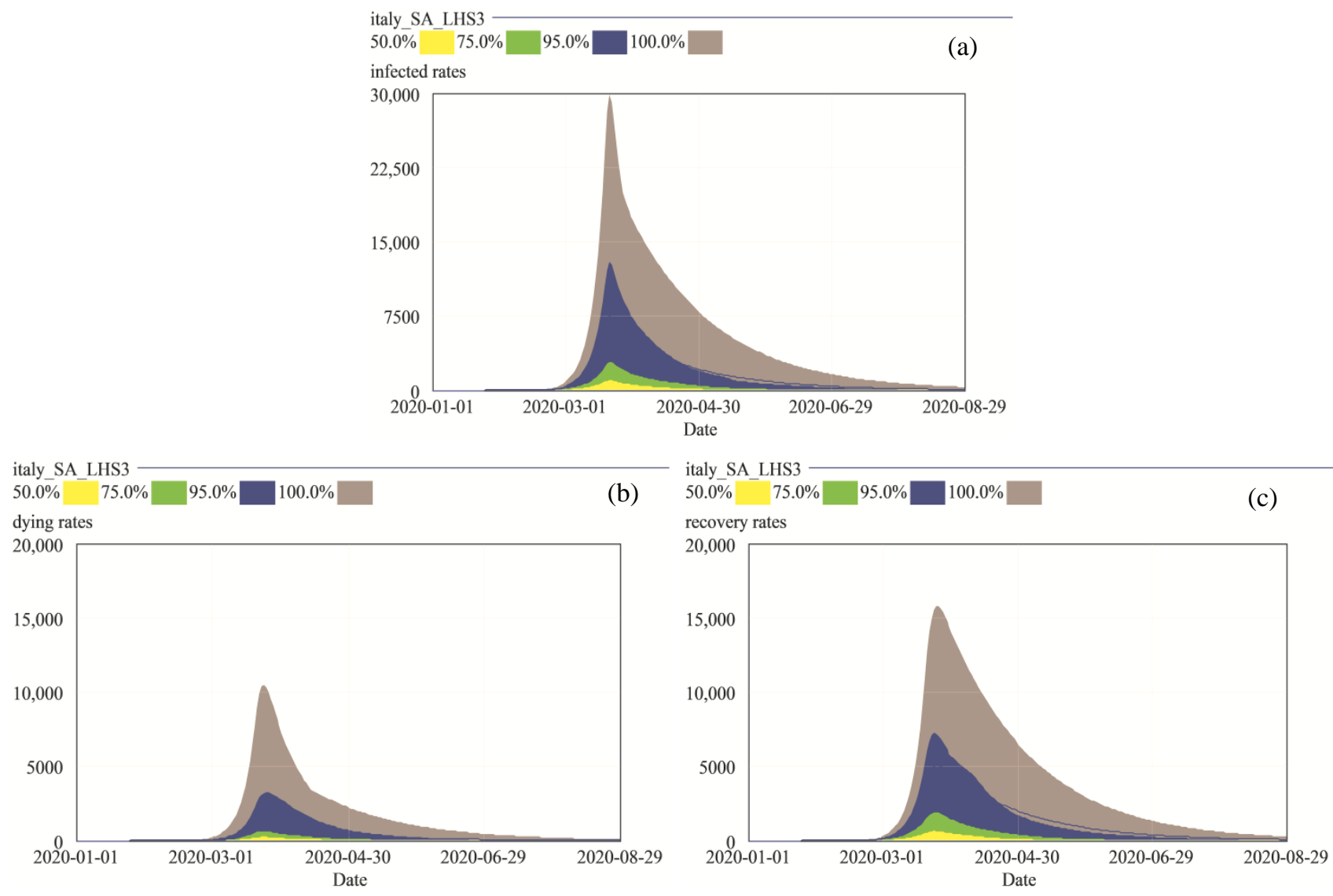

Figure A2. The sensitivity analysis based on LHS. (a) Infected rates; (b) Death rates; (c) Recovered rates 
Figures A2 show that the peak of infected, death, and recovered cases lies within the $95 \%$ of the total sampling of the sensitivity analysis. For instance, Figure 1 informs us that the peak of observed infected cases during the first wave was about 8,000 (people/day) which is in the range of the $95 \%$ sampling of infected cases. Please be noticed that the 95\% sampling of infected cases (Figure A1a) ranges between 3,500 and 11,000 (people/day).

Moreover, the sensitivity analysis does not only enable us to obtain highly sensitive parameters but also reminds us to focus on highly sensitive parameters. If we can control these highly sensitive parameters, this means that we can minimize the flow of pandemic or the flow of infectious diseases. 